\title{
Photometry of the five marginally studied open clusters Collinder 74, Berkeley 27, Haffner 8, NGC 2509, and VdB-Hagen $4^{\star}$
}

\author{
G. Carraro ${ }^{1,2}$ and E. Costa ${ }^{1}$ \\ 1 Departamento de Astronomía, Universidad de Chile, Casilla 36-D, Santiago, Chile \\ e-mail: giovanni.carraro@unipd.it \\ 2 Dipartimento di Astronomia, Università di Padova, Vicolo Osservatorio 2, 35122 Padova, Italy
}

Received 5 September 2006 / Accepted 15 November 2006

\section{ABSTRACT}

\begin{abstract}
Context. The stellar populations in the outer Galactic disk are a subject of wide interest nowadays. To contribute to a better picture of this part of the Galaxy, we have studied the nature of five marginally investigated star clusters (Collinder 74, Berkeley 27, Haffner 8, NGC 2509, and VdB-Hagen4) by means of accurate CCD photometry in the $V$ and $I$ passbands. These clusters are in fact located in the third Galactic quadrant.

Aims. We aim to obtain the basic parameters of these objects, which in some cases are still disputed in the literature. In the case of VdB-Hagen 4 we provide the first estimate of its fundamental parameters, while for Haffner 8 we present the first CCD photometry. Methods. The analysis is based on the comparison between field stars decontaminated color magnitude diagrams and stellar models. Particular care is devoted to the the assessment of the data quality and the statistical field star decontamination. The Padova library of stellar isochrones is adopted in this study.

Results. The analysis we carried out allowed us to solve a few inconsistencies in the literature regarding Haffner 8 and NGC 2509. Collinder 74 is found to be significantly older than reported before. VdB-Hagen 4 is a young open cluster located more than $20 \mathrm{kpc}$ from the Galactic center. Such an extreme distance is compatible with the cluster belonging to the Norma-Cygnus arm.
\end{abstract}

Key words. open clusters and associations: general, stars: Hertzsprung-Russell (HR) and C-M diagrams

\section{Introduction}

The main reason to acquire and analyze photometry of star clusters in the Galactic disk resides in the possibility of building up their color magnitude diagram (CMD), which allows us to infer estimates of the cluster age, distance, and reddening. In turn, these parameters are routinely used to trace the large scale structure and evolution of the Galactic disk. In particular, the outer Galactic disk is a topic of wide interest nowadays, with the discovery of new spiral features (Moitinho et al. 2006a), and the intensely studied structure of the outer disk, where the warp and flare seem to play a major role (Momany et al. 2006), but where at the same time external objects in the act of engulfing the Milky Way like the Monoceros Stream (Newberg et al. 2002) are claimed to have been detected. In this context stars clusters are well recognized to be valuable tracers of the disk structure, such that robust estimates of their fundamental parameters are necessary to proceed in our understanding of the details of the Galaxy structure.

In an attempt to contribute to all this, we present here CCD photometry of 5 Galactic fields, which contain the star clusters Collinder 74, Berkeley 27, Haffner 8, NGC 2509, and VdBHagen 4. These clusters are all located in the third Galactic quadrant, spanning 50 degrees in longitude and 15 in latitude (see Table 1). Some of these clusters (Collinder 74, Berkeley 27, Haffner 18 and NGC 2509) have already been investigated in the past at different levels, and for them we are going to

* Photometric data is only available in electronic form at the CDS via anonymous ftp to cdsarc.u-strasbg.fr $(130.79 .128 .5)$ or via http://cdsweb.u-strasbg.fr/cgi-bin/qcat?J/A+A/464/573
Table 1. Coordinates of the clusters under investigation for J2000.0 equinox, which we visually verified.

\begin{tabular}{ccccc}
\hline \hline Name & RA & Dec & $l$ & $b$ \\
\hline & hh:mm:ss & ${ }^{\circ}:^{\prime}:^{\prime \prime}$ & {$[\mathrm{deg}]$} & {$[\mathrm{deg}]$} \\
\hline Collinder 74 & $05: 48: 30$ & $+07: 24: 00$ & 198.97 & -10.41 \\
Berkeley 27 & $06: 51: 18$ & $+05: 46: 00$ & 207.78 & +02.60 \\
Haffner 8 & $07: 23: 24$ & $-12: 20: 00$ & 227.53 & +01.34 \\
NGC 2509 & $08: 00: 48$ & $-19: 03: 06$ & 237.85 & +05.82 \\
VdB-Hagen 4 & $07: 37: 44$ & $-36: 04: 00$ & 249.96 & -07.10 \\
\hline
\end{tabular}

provide a close comparison of the data and the derived results, since in the literature there still exist some discrepancy in their basic parameters.

On the other hand, VdB-Hagen 4 was never studied before. Still, it seems a very promising target, since it is located (see Table 1) very close to the center of the much discussed Canis Major over-density (Momany et al. 2006).

The paper is organized as follows. In Sect. 2 we detail the observations and reduction of the material that is the base of the present study. Section 3 illustrates the method adopted to estimate the radius of each cluster. In Sect. 4 we deal with field star decontamination before deriving the clusters basic parameters in Sect. 5. Section 6 summarizes the findings of this paper, suggesting some directions for future investigation.

\section{Observation and data reduction}

$V, I$ images centered on the star clusters Collinder 74, Berkeley 27, Haffner 8, NGC 2509, and VdB-Hagen 4 were 
obtained with the Cerro Tololo Inter-American Observatory $0.9 \mathrm{~m}$ and $1.0 \mathrm{~m}$ telescopes, which are operated by the SMARTS $^{1}$ consortium.

\section{1. $0.9 \mathrm{~m}$ observations}

Collinder 74, Berkeley 27, Haffner 8, and NGC 2509 were observed with the $0.9 \mathrm{~m}$ telescope on the nights of December 3 and 4, 2005. The pixel scale of the $2048 \times 2046$ Tek $2 k \#$ CCD is 0.369 , yielding a field of $13.5 \times 13.5$ arcmin on the sky. Both nights were photometric with average seeing of 1.1 arcsec. Due to the presence of very bright stars, Berkeley 27 and NGC 2509 were not placed in the center of the CCD (see Figs. 2 and 4). To secure a photometric calibration, the Landolt (1992) standard fields SA 98, PG 0231, TPhenix, G97, and G102 were observed at different air masses several times over the nights. The photometric solutions of the two nights were identical within the errors, so we derived a single photometric solution for the two nights, using the whole set of 170 standard stars. The calibration equations have the form:

$v=V+(2.001 \pm 0.003)+(0.16 \pm 0.01) \times X+(0.007 \pm 0.002) \times(V-I)$ $i=I+(2.898 \pm 0.007)+(0.08 \pm 0.01) \times X+(0.031 \pm 0.004) \times(V-I)$,

and the final rms of the fitting turned out to be 0.020 and 0.022 for the $V$ and $I$ passbands, respectively.

\subsection{0 m observation}

The star cluster Vdb-Hagen 4 was observed with the $1.0 \mathrm{~m}$ telescope on the night of December 2, 2005. The telescope is equipped with a new $4 \mathrm{k} \times 4 \mathrm{k} C \mathrm{CD}$ camera having a pixel scale of $0.289 /$ pixel which allows one to cover a field of $20^{\prime} \times 20^{\prime}$. Three Landolt (1992) areas (TPhoenix, Rubin 149, and PG 0231+006) were also observed to transform the instrumental magnitudes into the standard system. The night was photometric with an average seeing of 1.1 arcsec. In this case, the photometric solution for a grand-total of 69 stars reads:

$v=V+(1.934 \pm 0.004)+(0.16 \pm 0.01) \times X+(0.014 \pm 0.005) \times(V-I)$ $i=I+(2.786 \pm 0.008)+(0.08 \pm 0.01) \times X-(0.012 \pm 0.008) \times(V-I)$,

and the final rms of the fitting turns out to be 0.025 and 0.030 for the $V$ and $I$ passbands, respectively. Due to the presence of a very bright star, the center of the frame was chosen about 10 arcmin southward of the cluster. The covered areas are shown in Figs. 1 to 5, while Table 2 contains the observational log-book.

The data was reduced using the IRAF $^{2}$ packages CCDRED, DAOPHOT, and PHOTCAL. Photometry was done following the point spread function (PSF) method (Stetson 1987). The run of photometric errors derived from the PSF fitting is shown in Fig. 6. Global photometric errors have been estimated using the scheme developed by Patat \& Carraro (2001), which takes into account the errors coming from the PSF fitting procedure (ALLSTAR) and the calibration errors (corresponding to the zero points, color terms, and extinction). It turns out that stars brighter than $V \approx 20$ mag have global photometric errors (DAOPHOT internal plus calibration errors) lower than $0.25 \mathrm{mag}$ in magnitude and lower than $0.35 \mathrm{mag}$ in color. The final catalog consists of $2198,4632,4746,4835$, and 8353 point

\footnotetext{
1 http://www . astro.yale. edu/smarts/.

2 IRAF is distributed by NOAO, which are operated by AURA under cooperative agreement with the NSF.
}



Fig. 1. $I=600 \mathrm{~s}$ image of Collinder 74. The field is $13.5 \mathrm{arcmin}$ on a side. North is up, East to the left.

sources for Collinder 74, Berkeley 27, Haffner 8, and NGC 2509 and VdB-Hagen 4, respectively. The photometry was finally aperture-corrected filter by filter, using a suitable number (typically 15-20) of bright stars in the field. The correction varied between 0.080 and 0.210 mag.

Finally, the completeness corrections were determined by artificial star experiments on our data. Basically, we created several artificial images by adding artificial stars to the original images. About 1000-5000 - depending on the frame - were added to the original images. In order to avoid the creation of overcrowding, in each experiment we added only $15 \%$ of the original number of stars at random positions. The artificial stars had the same color and luminosity distribution of the original sample. In this way we found that the completeness level is better than $50 \%$ down to $V=20.5$.

\section{Clusters size}

We used star counts to estimate the size of each cluster under study. The center of each cluster is searched for by inspecting Figs. 1 to 5. In the case of VdB-Hagen 4 and Berkeley 27, the clusters are offset with respect to the center of the frame, due to the presence of very bright stars, which would not allow us to observe the fainter stars of the cluster. Star counts are performed by counting the number of stars in concentric rings 0.5 arcmin wides around the cluster center, and dividing by the ring surface. In the case of $\mathrm{VdB}$-Hagen 4 we employed rings 0.25 arcmin wide.

To minimize absorption effects we have employed the $I$ band, the same we used in the series of Figs. 1 to 5 . The aim of this procedure is to try to pick up as many cluster members as possible limiting the contamination of field stars. We consider the distance from the center at which the star counts keep flatas an estimate of cluster radius, thus meaning that the level of the field has been reached.

The results are shown in the various panels of Fig. 7. Examining this figure, we can conclude that:

1. all the clusters are well confined within the observed region; 


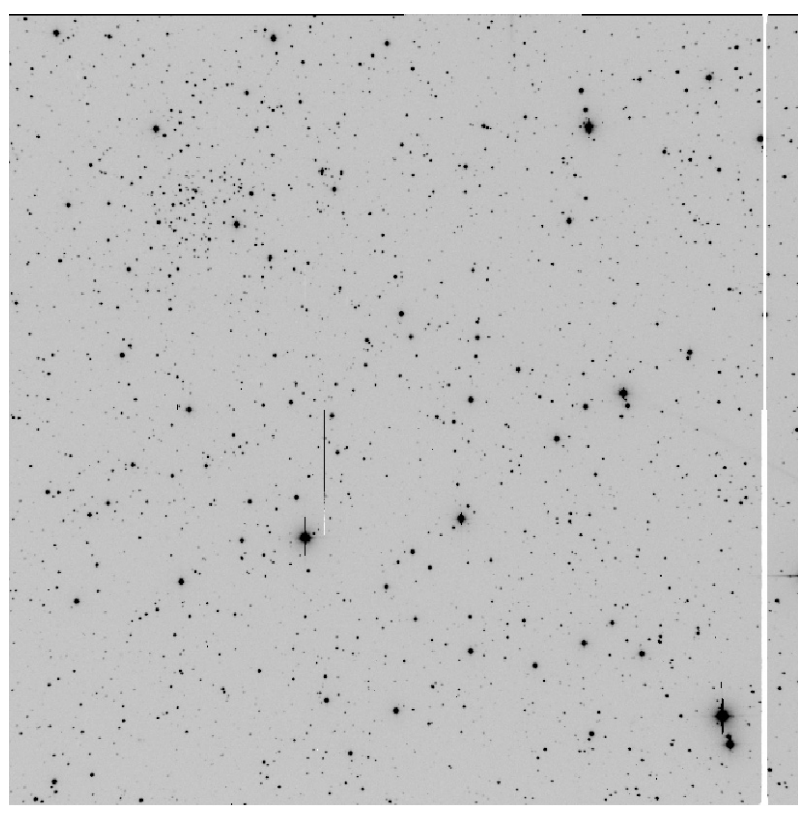

Fig. 2. $I=600 \mathrm{~s}$ image of Berkeley 27. The field is $13.5 \operatorname{arcmin}$ on a side. North is up, East to the left.



Fig. 3. $I=600 \mathrm{~s}$ image of Haffner 8 . The field is $13.5 \mathrm{arcmin}$ on a side. North is up, East to the left.

2. Collinder 74 is the cluster with the largest radius, amounting to 4.0 arcmin; on the other hand, it is the closest cluster, too;

3 . all the other clusters are smaller, with radii in the range between 1.0 (VdB-Hagen 4) and 3 (Berkeley 27) arcmin.

These estimates nicely compare with Dias et al. (2001) values of the clusters' diameters, which are based on visual inspection. The values of the radii are reported in Table 3. Having derived an estimate of the cluster radius, we can proceed with realizations of each cluster population and nearby Galactic fields.

\section{Field star decontamination}

Open star clusters in the Galactic disk are known to suffer from field star contamination that, depending on the cluster location inside the disk, can make it very difficult to derive the cluster

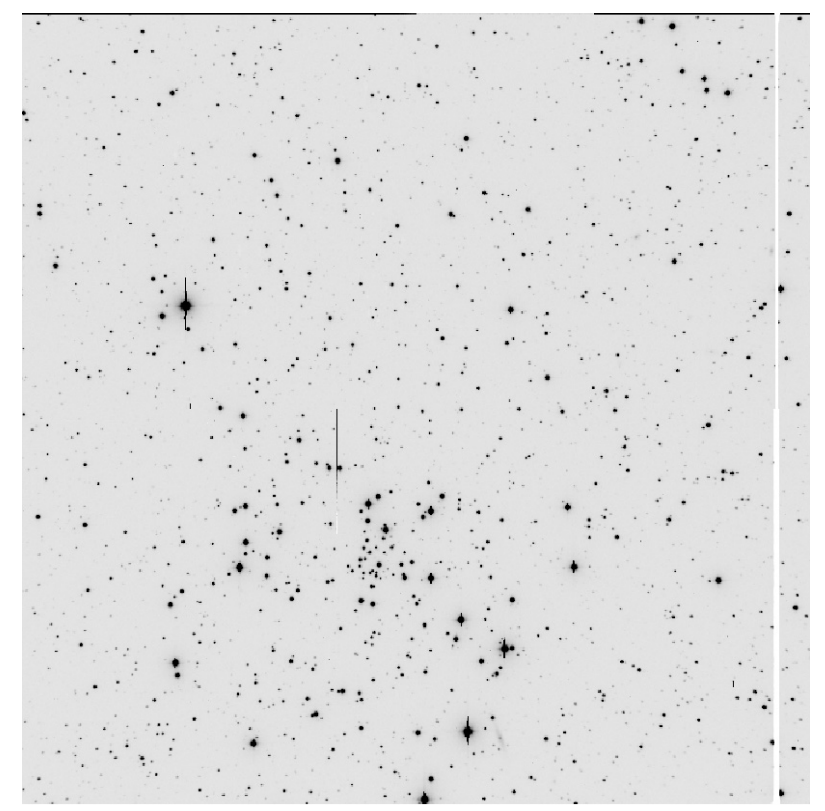

Fig. 4. $I=600 \mathrm{~s}$ image of NGC 2509 . The field is $13.5 \operatorname{arcmin}$ on a side. North is up, East to the left.

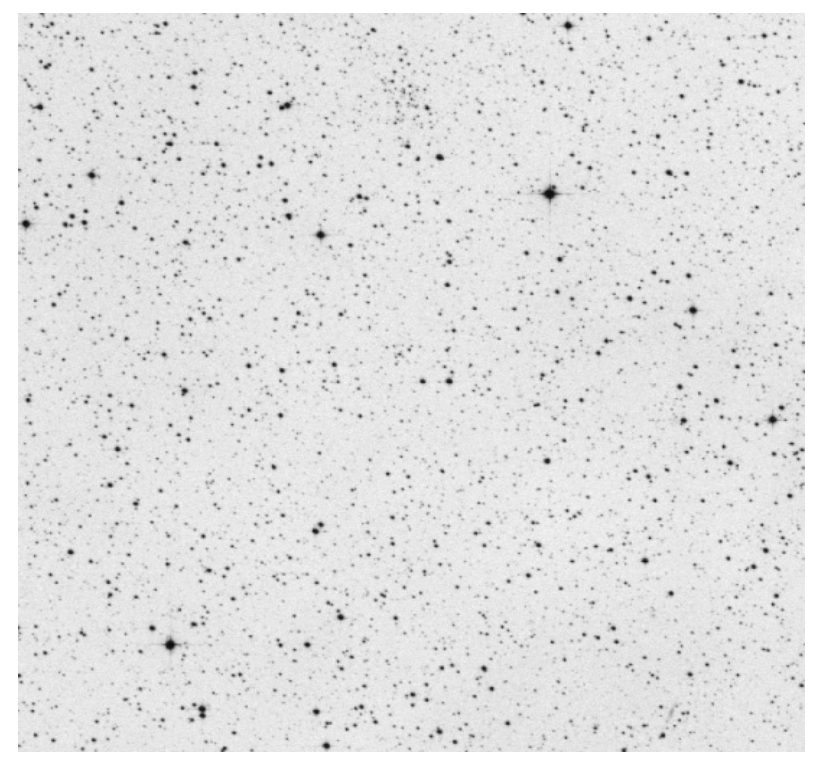

Fig. 5. $I=900 \mathrm{~s}$ image of VdB-Hagen 4. The field is $20 \operatorname{arcmin}$ on a side. North is up, East to the left.

fundamental parameters and in some cases even to assess the cluster reality. Here we make use of the results of the previous Sect. to obtain a realization of the field star population in the direction of each cluster, and then to statistically clean the cluster CMD. This way we can better assess the reality of the cluster, and, in positive cases, proceed to determine its fundamental parameters. Star counts performed in the previous section are already suggestive of the reality of the clusters under investigation. However, differential reddening plays a role in producing artificial clumps of stars, which look like real clusters. The star distribution in the CMD should help to get a clearer view of what is going on in the Galactic directions we are looking at in this paper.

In the series of Figs. 8 to 12, for each cluster we show the CMD of the stars enclosed in a circular region having the radius derived in Sect. 3 (left panels), the CMD of an off-set field 
Table 2. Log of photometric observations on December 2, 3, and 4, 2005.

\begin{tabular}{lcrr}
\hline \hline Cluster & Date & Filter & Exp time (s) \\
\hline VdB-Hagen 4 & 02 December 2005 & $V$ & $10,180,900$ \\
& & $I$ & $5,180,900$ \\
Collinder 74 & 03 December 2005 & $V$ & $10,20,600$ \\
& & $I$ & $5,2 \times 10,600$ \\
Haffner 8 & 03 December 2005 & $V$ & $10,20,600$ \\
& & $I$ & $5,10,600$ \\
NGC 2509 & 04 December 2005 & $V$ & $10,20,600$ \\
& & $I$ & $5,10,600$ \\
Berkeley 27 & 04 December 2005 & $V$ & $10,20,600$ \\
& & $I$ & $5,10,600$ \\
\hline
\end{tabular}



Fig. 6. Photometric errors in $V$ and $(V-I)$ as a function of $V$ mag.

outside the cluster region (middle panels), and the CMD of the cluster after having statistically removed the field stars (right panels). We stress the fact that the off-set fields have the same area of the cluster fields. Besides, in the particular cases of Berkeley 27 and VdB-Hagen 4, we applied the technique using more than one field realization.

The statistical technique we employ works as follows. We pick up an offset field star and look in the cluster CMD for the closest star in color and magnitude, and remove this star from the cluster star list. This procedure is repeated for all the offset field stars.

As amply discussed in the literature (see for instance Gallart et al. 2003; and Bertelli et al. 2003), from which our code has been designed, the method depends on two crucial parameters: the size of the searching ellipse, and the completeness of the photometry. The completeness is known (see Sect. 2), although in this particular case is not a big issue, since cluster and offset field come from the same frame and therefore suffer from the same amount of incompleteness. The size of the searching ellipse (see Gallart et al. 2003) has to be fixed case by case by running many trials.

\subsection{Collinder 74}

This appears a sparse cluster (see Fig. 1) defined by a handful of bright stars (Collinder 1933). Its low Galactic latitude is remarkable, and would mean the cluster is nearby, if real. The results of the cleaning procedure are shown in Fig. 8. With respect to the equal area field in the middle panel, the cluster realization in the left panel shows a narrower Main Sequence (MS), and the handful of bright stars we mentioned above. The clean CMD in the



Fig. 7. Estimating the clusters' radius through star counts.

right panel shows a MS that extends down to $V \sim 19.5$. However, it might be difficult to interpret the distribution of the bright red stars and to clearly define the cluster turn off point (TO).

\subsection{Berkeley 27}

This looks like a faint cluster (see Fig. 2). The CMD in the left panel of Fig. 9 exhibits a MS more populated than in the equal area field realization in the middle panel. The clean CMD shows a clear MS extending down to $V \sim 20.5$, with a TO located a $V \sim 17.0$. The bunch of bright red $((V-I) \geq 1.5)$ stars visible in the left panel are very probably cluster stars. We conclude that Berkeley 27 is undoubtedly a real cluster.

\subsection{Haffner 8}

Haffner 8 is a sparse open cluster very similar in structure to Collinder 74 (see Fig. 3). However, the clean CMD (see Fig. 10, right panel) reveals a much younger cluster, with only a few evolved stars. The shape of the MS if a typical one for star clusters a few hundreds million years old.

The TO point is located at $V \sim 13.5$ and the MS is well populated down to the limiting magnitude.

\subsection{NGC 2509}

The cluster is small but prominent (see Fig. 4), and seems to harbor a significant number of bright stars. The clean CMD in Fig. 11 (right panel) shows a remarkable clump of bright stars typical of a star cluster of the Hyades age (about 600 Myr). The shape of the clump closely resembles that of clusters like NGC 2635 (Moitinho et al. 2006b), an intermediate age open cluster located in the third Galactic quadrant as well. The TO region is also very well defined, with the TO at $V \sim 14.0$. The color of the TO indicates that the cluster suffers from a small amount of reddening, which is compatible with its high Galactic latitude (see Table 1). The MS seems to terminate abruptly at $V \sim 18.5$, which again might indicate that some low mass stars evaporation already occurred.

\subsection{VdB-Hagen 4}

This cluster was never studied before, and was first detected by van den Bergh \& Hagen (1975). They describe the cluster as 
Table 3. Fundamental parameters as derived from isochrone fitting.

\begin{tabular}{ccccccccc}
\hline \hline Name & $\begin{array}{c}\text { Apparent radius } \\
\text { arcmin }\end{array}$ & $\begin{array}{c}\text { Absolute diameter } \\
\mathrm{pc}\end{array}$ & $\begin{array}{c}E(V-I) \\
\mathrm{mag}\end{array}$ & $\begin{array}{c}\left(V-M_{V}\right) \\
\mathrm{mag}\end{array}$ & $\begin{array}{c}d_{\odot} \\
\mathrm{kpc}\end{array}$ & $\begin{array}{c}Z \\
\mathrm{pc}\end{array}$ & $\begin{array}{c}d_{\mathrm{GC}} \\
\mathrm{kpc}\end{array}$ & $\begin{array}{c}\text { Age } \\
\mathrm{Myr}\end{array}$ \\
\hline Collinder 74 & $4.0 \pm 0.5$ & 3.5 & $0.35 \pm 0.05$ & $11.75 \pm 0.10$ & 1.50 & -270 & 9.90 & 3000 \\
Berkeley 27 & $3.0 \pm 0.5$ & 4.2 & $0.35 \pm 0.05$ & $14.30 \pm 0.10$ & 4.80 & 200 & 12.90 & 2000 \\
Haffner 8 & $2.5 \pm 0.5$ & 3.8 & $0.30 \pm 0.05$ & $12.80 \pm 0.10$ & 2.60 & 60 & 10.40 & 500 \\
NGC 2509 & $2.5 \pm 0.5$ & 4.2 & $0.08 \pm 0.05$ & $12.50 \pm 0.10$ & 2.90 & 200 & 10.30 & 1200 \\
VdB-Hagen 4 & $1.0 \pm 0.5$ & 11.2 & $0.45 \pm 0.05$ & $17.50 \pm 0.20$ & 19.30 & 2300 & 23.50 & $\sim 200$ \\
\hline
\end{tabular}

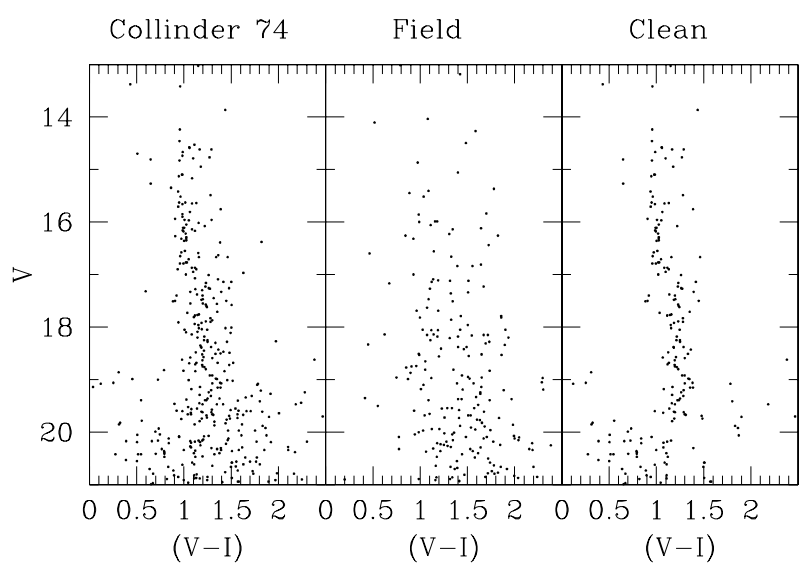

Fig. 8. Field star decontamination: Collinder 74.

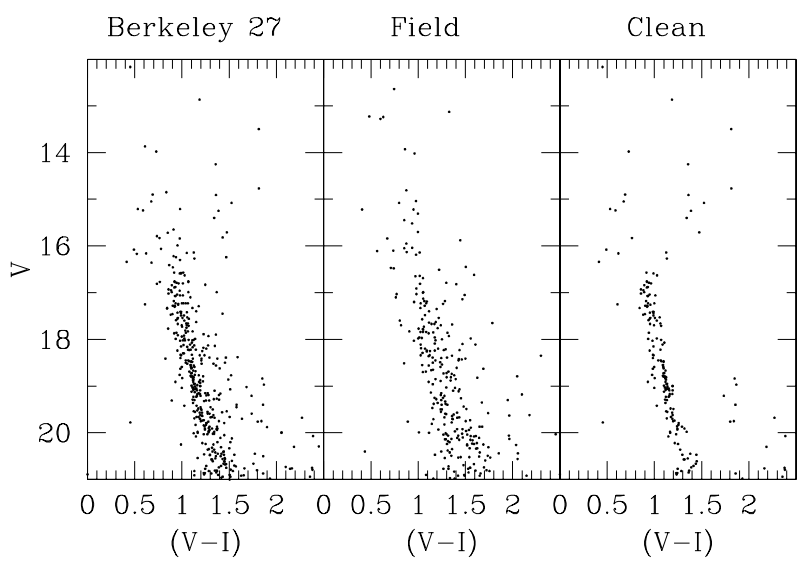

Fig. 9. Field star decontamination: Berkeley 27.

a poor ensemble of stars, but as a real feature both in the blue and in the red plates. This is confirmed by inspecting Fig. 5, where we have placed the cluster in the upper part of the frame due to the presence of a disturbing very bright star. Start counts confirm the cluster is small. The CMD of the cluster (left panel of Fig. 12) and the field (middle panel) look similar except for a tight blue sequence in the cluster area, which indicates the presence of a young cluster/association. Indeed, the clean CMD maintains this sequence weakening the field star contamination. The cluster MS is defined down to $V \sim 21$, where it intersects the field MS. VdB-Hagen 4 is more probably a faint distant star cluster. The contamination of field stars is really severe in this case, and it is not possible to firmly probe the presence of evolved stars.

\section{Cluster fundamental parameters}

We now use the results of previous section to infer estimates of the cluster fundamental parameters. For this purpose we shall

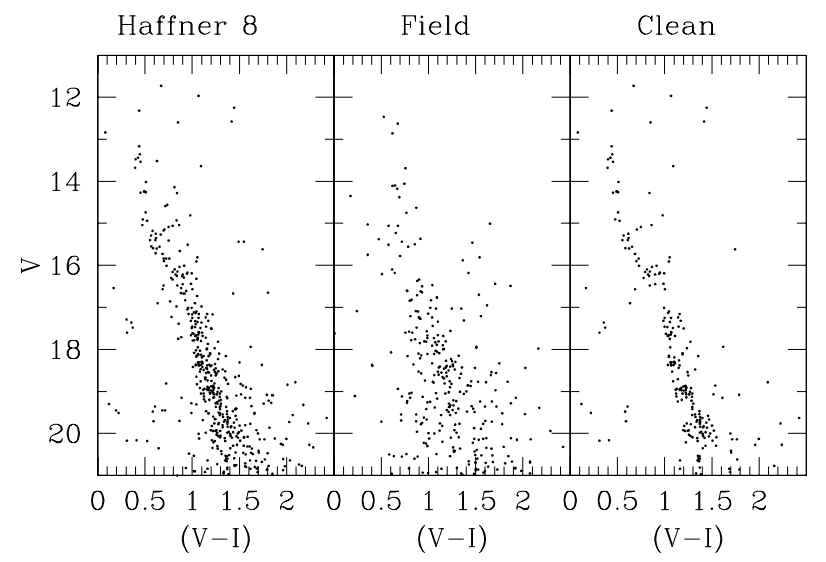

Fig. 10. Field star decontamination: Haffner 8.



Fig. 11. Field star decontamination: NGC 2509.

compare the clean CMDs with stellar isochrones from Girardi et al. (2000). Lacking any spectroscopic estimate of the metal abundance, we shall adopt the conservative solar metal abundance $Z=0.019$. The results are summarized in Table 3, where the radius, reddening, and distance modulus are reported together with their uncertainties. These reflect the degree of freedom we have in displacing an isochrone while still keeping a good fit. In addition, in the same table the heliocentric and Galactocentric distance are listed together with the best-fit age. Moreover, for each cluster we are going to recall any previous results and make a detailed comparison.

\subsection{Collinder 74}

This object was studied by Ann et al. (1999), who provide $U B V I$ photometry of a $11.5 \times 11.5$ field centered on the cluster. We compared our photometry with theirs, and found that $\Delta V=-0.072 \pm 0.049, \Delta(V-I)=-0.035 \pm 0.042$ (in the sense their study minus the present one) from 135 stars in common 


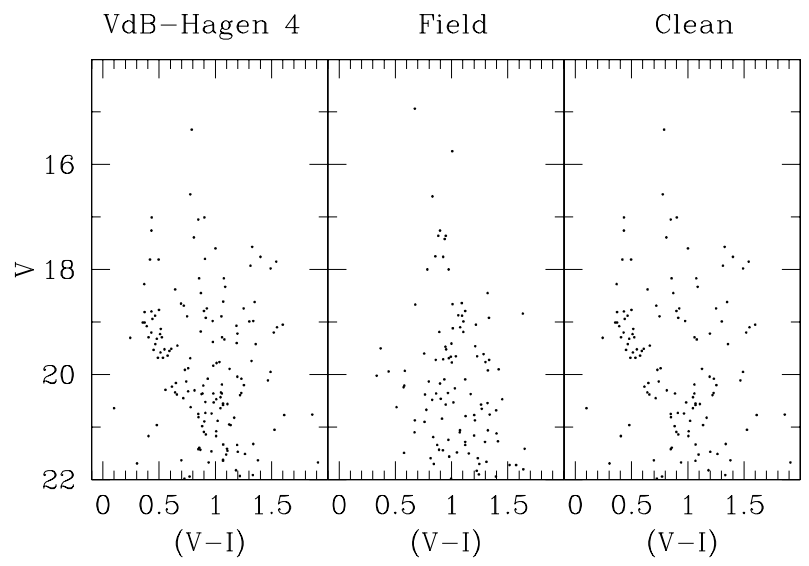

Fig. 12. Field star decontamination: VdB-Hagen 4.

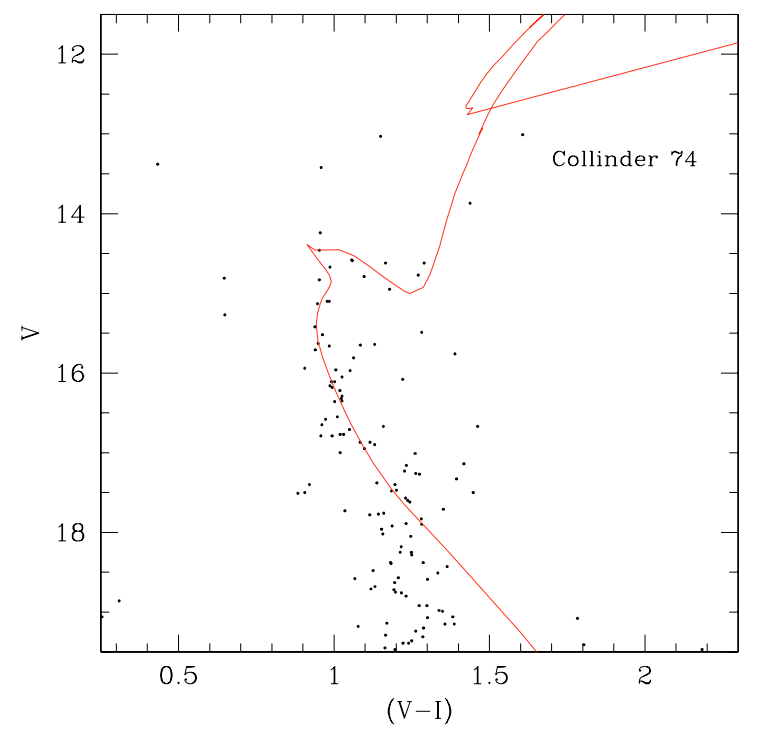

Fig. 13. Isochrone solution for Collinder 74. The $3 \mathrm{Gyr}$ isochrone is shifted by $E(V-I)=0.35$ and $V-M_{V}=11.75$.

brighter than $V=18.0$, and concluded that the two studies are largely consistent. These authors suggest that Collinder 74 is a star cluster 1.3 billion years old located at $1.5 \mathrm{kpc}$ from the Sun. The clean CMD is shown again in Fig. 13. Here a solar metallicity isochrone is superimposed to the candidate cluster members. After playing with several different ages, we come out with a best-fit age of $3 \mathrm{Gyr}$, which is shifted by $E(V-I)=0.35$ and $\left(V-M_{V}\right)=11.75$. By adopting a normal extinction law, where $\frac{E(V-I)}{E(B-V)}=1.245, E(V-I)=0.35$ turns into $E(B-V)=0.28$, which implies a heliocentric distance of $1.5 \mathrm{kpc}$.

The reason for which we adopted this fit resides in the nice reproduction of the TO region. As a consequence, the bulk of bright red stars mentioned above are interpreted as sub-giant and giant branch stars. A couple of brighter and redder stars cannot be fitted, and might mean that the cluster is metal richer, in case they were members. The MS seems to abruptly drop at $V \sim 18$, which is compatible with the cluster having lost most of its lowmass stars.

As guessed in the previous section, the cluster is located close to the Sun. Its position and height above the Galactic plane $(Z=270 \mathrm{pc})$ are reasonable for a cluster of this age. We believe that the significantly older age we found with respect to previous investigations is due to the better treatment of the field star contamination, which allows the various features of the CMD to

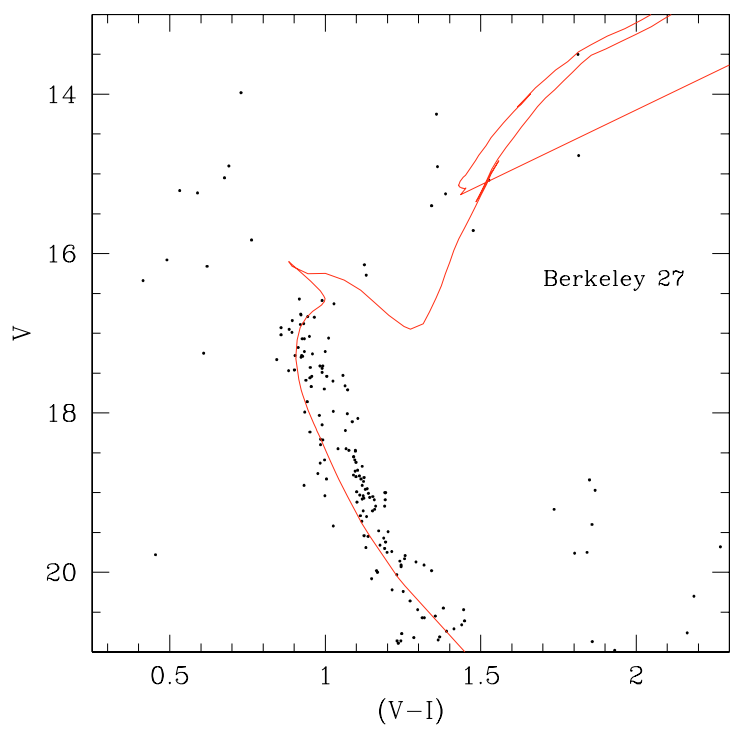

Fig. 14. Isochrone solution for Berkeley 27. The $2 \mathrm{Gyr}$ isochrone is shifted by $E(V-I)=0.35$ and $V-M_{V}=14.30$.

stand out more clearly. The lack of any red giant branch (RGB) clump is not unusual for a cluster of this age, since several other old clusters are known to have a few clump stars, if any (Phelps et al. 1994).

\subsection{Berkeley 27}

Berkeley 27 (Setteducati \& Weaver 1962) is also known as Biurakan 11. The only previous investigation we are aware of is by Hasegawa et al. (1999). They obtained $B V I$ photometry with of a 5':1 × 5'1 field centered on the cluster. Their analysis leads to these values for the cluster parameters: an age of 2.0 billion years, a distance of $13.5 \mathrm{kpc}$ from the Galactic center, and a reddening $E(V-I)=0.30$. The result of our fit is shown in Fig. 14. We basically find the same overall solution proposed by Hasegawa et al. (1999). We confirm that the probable age is around $2.0 \mathrm{Gyr}$, and the corresponding isochrone has to be shifted by $E(V-I)=0.35$ and $\left(V-M_{V}\right)=14.30$. This in turn yields a heliocentric distance of $4.8 \mathrm{kpc}$, and a height above the Galactic plane $Z \sim 250$ pc. With Collinder 74 this cluster shares the lack of a clear RGB and clump, but exhibits a wider MS, probably due some uncorrected field star contamination, a probable percentage of unresolved binaries, and some differential reddening. This latter might in fact plays a more important role than in the case of Collinder 74, due to the lower Galactic latitude of Berkeley 27.

\subsection{Haffner 8}

For this cluster only the old photographic study by Fenkart et al. (1972) is available. These authors suggest the cluster is 500 million year old, and it is located at $1.7 \mathrm{kpc}$ from the Sun. Strangely, they assign zero absorption to the cluster, which one would not expect judging from the location of the cluster relatively low on the Galactic plane. In fact, for instance, from Schlegel et al. (1998) maps, one can infer $E(B-V)=0.66$, which would imply $A_{V}=2.0$ at infinity, by assuming the normal extinction law $R_{V}=\frac{A_{V}}{E(B-V}=3.1$. Zero reddening would mean the cluster lies very close to the Sun. However, most probably something occurred wrong with the photographic photometry. Unfortunately, 


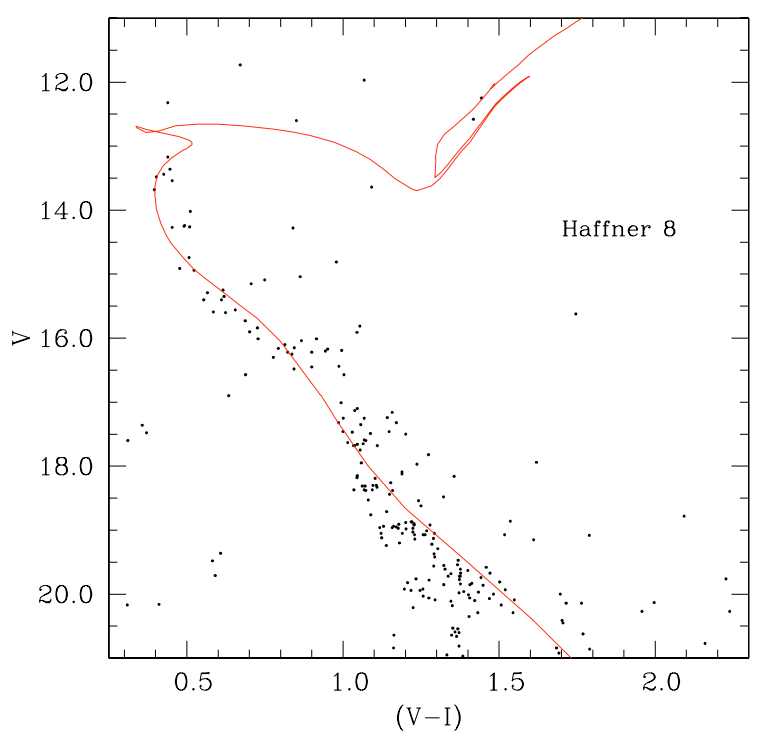

Fig. 15. Isochrone solution for Haffner 8 . The $0.5 \mathrm{Gyr}$ isochrone is shifted by $E(V-I)=0.30$ and $V-M_{V}=12.80$.

we could only compare the $V$ filter with them, and found that $\Delta V=-0.16 \pm 0.21$ for 32 stars in common brighter than $V=15$.

Our clean CMD is presented in Fig. 15. The $500 \mathrm{Myr}$ isochrone that provides the best fit is shifted by $E(V-I)=0.30$ and $V-M_{V}=12.80$. By assuming the normal extinction law, we get $E(B-V)=0.24$, which is reasonable for a cluster placed relatively low onto the Galactic plane. We point out that the reddening at infinity in this direction is $E(B-V)=0.66$ (Schlegel et al. 1998). The fit is very good, both in the TO region and along the MS. Also, the evolved part of the CMD is nicely reproduced. The derived parameters for Haffner 8 are listed in Table 3 .

\subsection{NGC 2509}

This cluster has been studied several times in the last few years, but different analyses led to very discrepant results. While Sujatha \& Babu (2003) found that the cluster is very old (8 Gyr), Ahumada (2000) concluded that NGC 2509 is only 1 Gyr old and has $E(B-V)=0.22$. The recent study by Tadross (2005) based on 2MASS does not solve the problem since it is not clear if the author assigns the age of 1.6 or $6.1 \mathrm{Gyr}$ to the cluster. Moreover, the same author assigns a suspicious value of $E(B-V)=0.0$ to the cluster. Even the reddening found by Ahumada does not seem correct, since the Schlegel et al. (1998) maps predict an absorption of $E(B-V)=0.16$ in this direction. Since this is the reddening value at infinity, NGC 2509 should be less reddened. However, the visual inspection of the cluster CMD overall favors the results of Ahumada (2000). In fact, the photometry by Sujatha \& Babu (2003) is highly suspicious, as was already found in the recent study of NGC 2401 (which they observed the same night as NGC 2509) by Baume et al. (2006). Our isochrone solution is illustrated in Fig. 16. Here the $1.2 \mathrm{Gyr}$ isochrone for solar metallicity has been shifted by $E(V-I)=0.08$ and $V-M_{V}=12.50$. While the TO region and the whole MS is nicely reproduced, this isochrone fails to reproduce the color of the RGB and clump. This may be due to the fact that the exact metallicity is not solar, an issue that can be solved only by a spectroscopic study of the clump stars. On the other hand, the mixing length parameter is an important ingredient of stellar evolutionary models that play a role in determining the precise color of RGB stars. For instance, the discussion in



Fig. 16. Isochrone solution for NGC 2509. The $1 \mathrm{Gyr}$ isochrone is shifted by $E(V-I)=0.08$ and $V-M_{V}=12.50$.



Fig. 17. Isochrone solution for $\mathrm{VdB}-$ Hagen 04 . The $0.2 \mathrm{Gyr}$ isochrone is shifted by $E(V-I)=0.45$ and $V-M_{V}=17.50$.

Palmieri et al. (2002) clearly demands a revision of the $(V-I)$ versus temperature relation, which may help to solve the discrepancy between theory and observations also in a metallicity regime higher than that of globular clusters.

\subsection{VdB-Hagen 4}

In Fig. 17 we show the CMD of VdB-Hagen 4 corrected for field star contamination. We superpose an isochrone of $200 \mathrm{Myr}$ shifted by $E(V-I)=0.43(E(B-V)=0.32)$ and $\left(V-M_{V}\right)=$ 17.50. As for the reddening, the value at infinity derived from Schlegel et al. (1998) maps is $E(B-V)=0.49$. The fit here is mainly based on the shape of the MS, and looks reasonable. This is confirmed by the empirical zero-age main sequence (ZAMS, dashed line) from Schmidt-Kaler (1982), which is displayed is the same plot and which has been shifted by the same apparent distance modulus and reddening. 
This fit demonstrates that the star clustering VdB-Hagen 4 is really extreme: it is a group of young stars located at about $19 \mathrm{kpc}$ from the Sun (see Table 3). This distance and the Galactic coordinates of the group are compatible with it being part of the outer (Norma-Cygnus) spiral arm (see Moitinho et al. 2006a). The height $Z$ above the Galactic plane is about $2 \mathrm{kpc}$, which is compatible with the maximum warping of the Galactic disk in the third Galactic quadrant (Carraro et al. 2006; Vázquez et al. 2006).

\section{Conclusions}

We have used new CCD photometry in the $V$ and $I$ passbands to study the stellar content of 5 Galactic fields containing the star clusters Collinder 74, Berkeley 27, Haffner 8, NGC 2509, and VdB-Hagen 4 . The basic parameters we estimated are summarized together with their uncertainties in Table 3. The most interesting results we found can be summarized as follows:

- Collinder 74 is found to be significantly older than in previous studies. With an age of $3 \mathrm{Gyr}$, it falls in an age interval where only a few old open clusters are known; therefore this cluster contributes to making less the claim that a burst of star formation occurred around 5 Gyr ago in the Galactic disk less important, as already found by Carraro et al. (2005).

- We clarify some discrepancies present in the literature for NGC 2509 and Haffner 8: the claim that NGC 2509 is very old and possesses zero reddening is found to have no observational support; the position of the RGB and the clump of NGC 2509 poses an interesting challenge for theoretical models, and more work is needed for this cluster, in particular a spectroscopic estimate of the metal content.

- The Haffner 8 zero reddening found by previous investigations is shown to be most probably due to problems in the calibration of the photographic photometry.

- As for Berkeley 27, this is the only cluster for which we find nice agreement with previous investigation .

- VdB-Hagen 4 is an extremely interesting small cluster; according to our analysis it is a young cluster belonging to the outer Galactic spiral arm in the extreme periphery of the Galactic disk. To better elucidate the stellar populations in this direction, $U B V$ photometry is needed.

- The cluster radii, expressed in parsec, are well within the typical radius of open star clusters in the Galactic disk (Janes et al. 1988). The only exception is VdB-Hagen 4, which has a significantly larger radius. This is, however, compatible with the trend of open cluster size as a function of the height from the Galactic plane (Chen et al. 2004).

With the exception of Haffner 8, the clusters of this sample exhibit heights above the Galactic plane larger than the typical Population I thin-disk scale height $(\approx 70 \mathrm{pc})$. We argue that this is probably an age effect, and in fact old open clusters (say older than the Hyades) do show on the average large heights above the Galactic plane (Friel 1995).

Acknowledgements. The work of G.C. is supported by a grant of the Departmento de Astronómia de Universidad de Chile. E.C. acknowledges FONDAP No. 15010003.

\section{References}

Ahumada, J. A. 2000, ASPC, 198, 43

Ann, H. B., Lee, M. G., Chun, M. Y., et al. 1999, JKAS, 32, 7

Baume, G., Moitinho, A., Vázquez, R. A., et al. 2006, MNRAS, 367, 1441

Bertelli, G., Nasi, E., Girardi, L., et al. 2003, AJ, 125, 770

van den Bergh, S., \& Hagen, G. L. 1975, AJ, 80, 11

Carraro, G., Geisler, D., Moitinho, A., Baume, G., \& Vázquez, R. A. 2005, A\&A, 442, 197

Carraro, G., Moitinho, A., Zoccali, M., Vázquez, R. A., \& Baume, G. 2006, AJ, in press

Chen, W. P., Chen, C. W., \& Shu, C. G. 2004, AJ, 128, 2306

Collinder, O. 1931, Ld.An., 2

Fenkart, R. P., Buser, R., Ritter, H., et al. 1972, A\&AS, 7, 487

Friel, E. D. 1995, ARA\&A, 33, 381

Gallart, C., Zoccali, M., Bertelli, G., et al. 2003, AJ, 125, 742

Girardi, L., Bressan, A., Bertelli, G., \& Chiosi, C. 2000, A\&AS, 114, 371

Hasegawa, T., Malasan, H. L., Kawakita, H., et al. 2004, PASJ, 56, 295

Janes, K. A., Tilley, C., \& Lynga, G. 1988, AJ, 95, 771

Landolt, A. U. 1992, AJ, 104, 372

Moitinho, A., Vázquez, R. A., Carraro, G., et al. 2006a, MNRAS, 368, L77

Moitinho, A., Carraro, G., Baume, G., \& Vázquez, R. A. 2006, A\&A, 445, 939

Momany, Y., Zaggia, S., Gilmore, G., et al. 2006, A\&A, 451, 515

Newberg, H. J., Yanny, B., Rockosi, C., et al. 2002, ApJ, 569, 245

Palmieri, R., Piotto, G., Saviane, I., Girardi, L., \& Castellani, V. 2002, A\&A, 292, 115

Patat, F., \& Carraro, G. 2001, MNRAS, 325, 1591

Phelps, R. L., Janes, K. A., \& Montgomery, K. A. 1994, AJ, 107, 1079

Sajatha, S., \& Babu, G. S. D. 2003, BASI, 31, 9

Schlegel, D. J., Finkbeiner, D. P., \& Davis, M. 1998, ApJ, 500, 525

Schmidt-Kaler, Th. 1982, Landolt-Börnstein, Numerical data and Funct ional Relationships in Science and Technology, New Series, Group VI, Vol. 2(b), ed. K. Schaifers, \& H. H. Voigt (Berlin: Springer Verlag), 14

Tadross, A. L. 2005, JKAS, 38, 357

Setteducati, A. F., \& Weaver, M. F. 1962, Newly Found Stellar Clusters (Berkeley: Radio Astr. Lab. University of California)

Stetson, P. B. 1987, PASP, 99, 191

Vázquez, R. A., May, J., Carraro, G., et al. 2006, ApJ, submitted 